\title{
Letter processing in the visual system: Different activation patterns for single letters and strings
}

\author{
KARIN H. JAMES and THOMAS W. JAMES \\ Indiana University, Bloomington, Indiana \\ and \\ GAEL JOBARD, ALAN C.-N. WONG, and ISABEL GAUTHIER \\ Vanderbilt University, Nashville, Tennessee
}

\begin{abstract}
One would expect that a lifetime of experience recognizing letters would have an important influence on the visual system. Surprisingly, there is limited evidence of a specific neural response to letters over visual control stimuli. We measured brain activation during a sequential matching task using isolated characters (Roman letters, digits, and Chinese characters) and strings of characters. We localized the visual word form area (VWFA) by contrasting the response to pseudowords against that for letter strings, but this region did not show any other sign of visual specialization for letters. In addition, a left fusiform area posterior to the VWFA was selective for letter strings, whereas a more anterior left fusiform region showed selectivity for single letters. The results of different analyses using both large regions of interest and inspections of individual patterns of response reveal a dissociation between selectivity for letter strings and selectivity for single letters. The results suggest that reading experience fine-tunes visual representations at different levels of processing. An important conclusion is that the processing of nonpronounceable letter strings cannot be assumed to be equivalent to single-letter perception.
\end{abstract}

Extensive experience with an object class can result in exquisite perceptual skills. Car experts can recognize at a glance the makes and models of hundreds of cars, and bird-watchers can identify the species of a bird briefly seen through foliage. The acquisition of such perceptual expertise with objects can lead to functional specialization within the brain systems dedicated to visual processing. Faces are commonly used to study the phenomenon of category-specific neural specialization (Kanwisher, 2000; Kanwisher, Chun, \& McDermott, 1996), but neural specialization has also been demonstrated for other expert object classes, including cars, birds, and even novel objects (e.g., "Greebles"; Gauthier, Tarr, Anderson, Skudlarski, \& Gore, 1999; Rossion, Gauthier, Goffaux, Tarr, \& Crommelinck, 2002). Most people are also perceptual experts with words. For adults, much of modern life is spent reading, and adult readers can recognize words with amazing efficiency (LaBerge \& Samuels, 1974). As children, most of us once dedicated a considerable amount of time to learning letters, and each day a literate person must process thousands of letters just to interact normally with the modern environment. Here, we hypothesize that

This research was supported by NEI Grant R01-EY13441 to I.G. and a grant from the James S. McDonnell Foundation to the Perceptual Expertise Network. The authors thank Danielle Brown, Debbie Boner, and Richard Baheza for technical assistance, and the members of the Perceptual Expertise Network for helpful discussions. Correspondence concerning this article should be addressed to K. H. James, Psychology Department, Indiana University, 1101 E. 10th Street, Bloomington, IN 47405 (e-mail: khjames@indiana.edu). this extensive experience results in letter-specific neural specialization of the visual system.

Most of the neuroimaging studies conducted with printed text were designed for the purpose of understanding the processes involved in reading. Therefore, it may not be surprising that there is only limited evidence speaking directly to the neural substrates involved in recognizing single letters. Several large circuits appear to underlie various aspects of reading. For example, a dorsal posterior system (angular gyrus, supramarginal gyrus, and superior temporal sulcus) is thought to subserve orthography-to-phonology correspondences (Black \& Behrmann, 1994). Semantic analyses of words are believed to occur predominantly in the left inferior frontal lobe and posterior superior temporal sulcus (Bookheimer, 2002; Gabrieli, Poldrack, \& Desmond, 1998), and context comprehension is considered a right hemisphere task (Kircher, Brammer, Tous, Williams, \& McGuire, 2001). A posterior ventral network including the occipitotemporal region is thought to underlie visual processing of printed text and to be responsible for the late-developing skills of rapid word recognition that result from increased reading experience (Frackowiak, Friston, Frith, Dolan, \& Mazziotta, 1997; Pugh et al., 2001). Within this system, the region that has received the most attention as a candidate area for early visual processing of printed text is the left fusiform gyrus.

Part of the left midfusiform gyrus shows higher activation for words than for consonant strings of the same length (Cohen et al., 2000; Dehaene, Le Clec'H, Poline, Le Bihan, \& Cohen, 2002) and has therefore been la- 
beled the visual word form area (VWFA; Talairach coordinates [TCs] $[x, y, z]$ approximately, $-42,-57,-15)$. However, the VWFA is also engaged by images of objects (Booth et al., 2002; Devlin et al., 2002; Moore \& Price, 1999; Murtha, Chertkow, Beauregard, \& Evans, 1999), which may not be surprising given its proximity to (and sometimes overlap with) the lateral occipital complex, an area defined as responding more to intact images of objects than to other visual stimuli (Malach et al., 1995). In addition, although the VWFA does not differentiate between pseudowords and words (Hagoort et al., 1999), it responds more to pseudowords than to consonant strings or "poorly constructed" pseudowords (McCandliss, Cohen, \& Dehaene, 2003). Therefore, the VWFA is thought to be involved in processing word-like stimuli, but its specific role remains controversial (for hypotheses, see Jobard, Crivello, \& Tzourio-Mazoyer, 2003; McCandliss et al., 2003; Price, 2000).

For our purposes, one important limitation of the reading neuroimaging literature is that studies rarely include comparisons between letter strings and nonletter controls. However, these comparisons are crucial for understanding early visual processing of print. In one of the first studies to compare letter strings with nonletter stimuli (Puce, Allison, Asgari, Gore, \& McCarthy, 1996), letter strings were compared with faces and textures, and an area (overlapping with the VWFA) selective for letter strings in the left occipitotemporal region was found (TCs $-40,-66,-17)$. In more recent studies, letter-stringsensitive regions that lie anterior and medial to the VWFA have been reported when letter strings are contrasted with digit strings (Polk \& Farah, 1998; Polk et al., 2002). In these studies, the location of the region that responded more to letter strings than to digit strings was highly variable among individuals (TC ranges: $x,-34$ to -51 ; $y,-19$ to $-67 ; z, 3$ to -16$)$. Other work has suggested a more posterior locus of activation for letter strings (TCs $-38,-90,-2$; Hasson, Levy, Behrmann, Hendler, \& Malach, 2002; Tagamets, Novick, Chalmers, \& Friedman, 2000). When faces were used as a baseline for letter strings, activation appeared posterior and ventral to the VWFA (TCs -37,-71,-22; Puce et al., 1996). Therefore, the location of a letter-string-sensitive area seems to be affected by the control category used: In comparison with activation for digit strings, that for letter strings was more anterior; when shapes or faces were used, the activation was more posterior. Although it is difficult to localize activation very precisely using magnetoencephalography, researchers using this technique also found evidence that a left inferior temporal region responds more to letter strings than to symbol strings (Tarkiainen, Helenius, Hansen, Cornelissen, \& Salmelin, 1999).

Our primary interest here is whether or not there is neural specialization for the perception of single letters. We initially learn letters in isolated form, but as adults we see them most frequently in the context of a word. Do we retain some neural specialization for the parts (letters) of the stimuli to which we are continually exposed (words)? To address this question, we must first consider which perceptual units we encode when reading. Intuitively, it seems that we would process the individual letters that make up a word, but some have argued that this is not the case. At very brief exposure durations, accuracy is poorer for single letters than for letters embedded in frequent words, an oft-cited finding referred to as the word superiority effect (Johnston \& McClelland, 1974; Reicher, 1969; Wheeler, 1970). After a brief exposure, observers can report several words but only about four letters (Cattell, 1886), and people will read a word correctly even if it is missing letters or if its letters are distorted (Pillsbury, 1897). Such findings have led to the idea that perhaps whole words are the perceptual units used when reading (see, e.g., McClelland, 1977; Monk \& Hulme, 1983). That is, common words may not be broken into smaller units. However, one argument against the notion that words can be read as wholes is that pseudowords (i.e., pronounceable nonwords) can be read faster than (unpronounceable) consonant strings. This implies that we can detect orthographic regularity, which requires breaking a word down into parts (Mewhort, 1974). In addition, some pseudowords can be read as fast as real words, suggesting that the word form of familiar words, if stored, does not speed up recognition (Baron \& Thurstone, 1973). These findings do not necessarily imply that each letter is processed; words may be broken down into two- or three-letter units or syllables. However, there is recent empirical evidence that each individual letter is processed as a word is read (Pelli, Farell, \& Moore, 2003). Using a word identification paradigm in which words are presented in Gaussian noise, Pelli et al. have shown that word identification efficiency is inversely proportional to word length. That is, individual letters must be identifiable in order for the word to be readable, and this holds true for even the most common three-letter words. In addition, the word superiority effect that initiated the modern idea of whole-word reading is seen only under special viewing conditions - that is, only when a backward pattern mask is used. Under normal viewing conditions, letters presented in isolation are identified faster than letters embedded in words (Johnston \& McClelland, 1974). Thus, although psychologists still debate about the conditions under which words are processed via letter identification, whether or not the differential processing of letters and words is reflected in the visual system has not been addressed directly.

Single letters have been used as stimuli in only a few neuroimaging studies. In one study, a lateral occipital region was found that showed adaptation upon repeated presentation of letters, but not upon repeated presentation of faces (Gauthier et al., 2000). Adaptation is often considered a strong test of a region's selectivity (GrillSpector et al., 1999). This region was just lateral to the midfusiform gyrus (bilaterally; TCs 53,-62,3). In three additional fMRI studies, single letters were compared to objects or symbols. Joseph, Gathers, and Piper (2003) found common letter and object activity as well as a 
trend toward a letter-specific area, both in the left fusiform gyrus. Flowers et al. (2004) found left occipital gyrus activation (BA 37) for letters over symbols and colors. In their study, attention to letters, symbols, and colors was manipulated, whereas visual activation to letters was not of primary concern. In a third study, Longcamp, Anton, Roth, and Velay (2003) found letter-sensitive regions in the precentral gyrus, left fusiform, and medial occipital regions. Thus, although only a few studies have been devoted to this question, there is some converging evidence that regions in the fusiform gyrus support the processing of isolated letters. However, with one exception (Longcamp et al., 2003), these studies do not compare letters directly to visually similar control items such as other types of characters, making it difficult to claim the area shows selectivity for letters.

Therefore, questions remain as to how and where letters are processed in the brain. It is possible that they are processed in the same regions of the brain as words and strings of letters. After all, letters are the parts from which words are constructed, and word processing has been thought to be part based (Farah, 1990). However, isolated letters may themselves be objects of expertise that do not elicit complex linguistic processing, and in that sense could be processed in similar regions as other objects of expertise. Although both letters and faces are objects on which we are experts, we would expect neural specialization for letters to differ from that observed for faces. According to the process-map hypothesis (Gauthier, 2000), the ventral occipital and temporal cortices are organized according to the type of processing that is performed on a stimulus. The visual processing that a given object requires may be determined by the recognition goals for that type of stimulus, combined with the information in the stimulus that can support this recognition goal. There are important differences between face expertise and letter expertise. Expertise in face recognition necessitates individuation of visually homogenous objects. Expertise in letter recognition requires inclusion of different objects in the same category (e.g., letters of different sizes or fonts; Wong \& Gauthier, 2002). An analysis of small differences would not be necessary for categorizing a letter, and although they may be analyzed (see van Leeuwen \& Lachmann, 2004), fine details do not define a letter's identity (Sanocki, Bowyer, Heath, \& Sarkar, 1998). Therefore, in general, global shape differences could be used to distinguish quickly among letters. For our present purposes, these are examples of differences that distinguish letter expertise from other types of object expertise that have been studied until now, and they suggest that letter expertise would engage a different neural substrate than face, car, or bird expertise. The question remains, however, as to whether isolated letters engage the same brain areas as words, pseudowords, or nonword letter strings.

In the present experiment, in addition to looking at specialization for single letters in the visual system, we also investigated the similarities and differences between the processing of character strings and that of single characters. We have included strings of characters and pseudowords so that we can compare our results with those of previous work on letter-string processing. Evidence to date has suggested that the left fusiform gyrus is involved in processing print stimuli, including letter strings (Hasson et al., 2002; Polk et al., 2002; Puce et al., 1996; Tagamets et al., 2000), pseudowords, words (Cohen et al., 2000), and possibly isolated letters (Joseph et al., 2003; Longcamp et al., 2003). Our results add to this research in several ways. First, we replicate previous work and show a putative VWFA that responds more to pseudowords than to letter strings. Second, we find an area posterior to the VWFA in the left fusiform gyrus that is selective for Roman letter strings over digit strings and Chinese character strings. These two areas are so near one another that they may be confused in cross-study comparisons, but they show dissociable patterns of response. Third, we find a more anterior area in the left fusiform gyrus that responds selectively to single Roman letters rather than single digits or Chinese characters. Most surprisingly, the area showing selectivity for strings is not selective for single characters, and vice versa. Moreover, a certain degree of selectivity for single letters appears to be more diffuse and distributed throughout the left fusiform gyrus, whereas selectivity for strings is more focal. These findings are supported by convergent ROI analyses and patterns of individual activation.

\section{METHOD}

\section{Subjects}

The subjects (4 females and 4 males) were graduate students or research assistants in the psychology department at Vanderbilt University. All gave informed consent according to the guidelines of the institutional review board of the Vanderbilt University Medical Center and were paid for their participation. All of the subjects were right-handed and reported normal or corrected-to-normal visual acuity and no history of neurological disorders. Their ages ranged from 20 to 42 years, with a median age of 25 years.

\section{Stimuli and Tasks}

All testing was conducted using Macintosh computers and RSVP software (www.cog.brown.edu/ tarr/RSVP). The stimuli were presented on two small LCD screens mounted within a VisuaStim XGA goggle system (MRI Devices, www.mrivideo.com) that was worn by the subject inside the scanner. The virtual sizes of the screens were $76.2 \times 57.2 \mathrm{~cm}$, and the screens were viewed from a virtual distance of $120 \mathrm{~cm}$. The sizes of each stimulus type are provided below.

The subjects were required to perform a 1-back sequential matching task. There were seven different stimulus types that were presented in various fonts: single Roman letters, digits, single Chinese characters, Roman letter strings, digit strings, Chinese character strings, and pseudowords. When the stimuli were single characters, the 1-back task was simply to match two sequentially presented images (same character, same font). During the strings blocks, the subjects performed the same 1-back task but only on the central character in the string (see Figure 1). The single characters and the central character of each string were underscored. No response was required on a nonmatch trial. The ratio of match to nonmatch trials was approximately $1: 8$. 
A

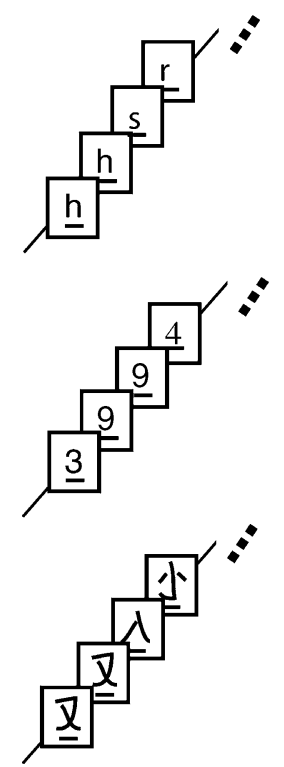

B
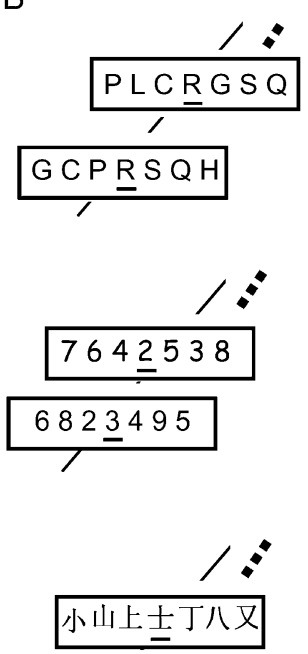

上山小士又八丁

Figure 1. Examples of (A) single characters, (B) strings of characters, and (C) pseudowords used in the experiment. On each trial, subjects had to decide whether or not the character on the screen matched the character that preceded it (1-back task). A response was required only on match trials (a go-no-go task). In the case of the character strings, the subjects matched only the underscored, central character. The Roman letters used were $H, L, S, R, G, P, C$, and Q; the digits were 2-9; and eight simple (four-stroke maximum) Chinese characters were presented for both the single and the string runs. Pseudowords also included the vowels $A, E$, and $U$. We attempted to avoid presenting common letter combinations within the pseudowords. The single characters subtended approximately $2.3^{\circ} \times 2.3^{\circ}$ of visual angle; the strings subtended $1.5^{\circ} \times 5.7^{\circ}$ of visual angle.

The single characters were in the center of the screen with their locations varying from trial to trial by about one half of a degree of visual angle around the center of the screen (jitter). The jitter was small enough that the central stimulus did not extend outside of foveal vision. The seven-character letter strings were constructed of seven of eight potential consonants presented in uppercase $(\mathrm{H}, \mathrm{L}, \mathrm{S}$, $\mathrm{R}, \mathrm{G}, \mathrm{P}, \mathrm{C}$, and Q) that were chosen in accordance with Polk et al.'s (2002) criteria for equating visual similarity between letter strings and digit strings. No consonant was repeated within a single string. These eight consonants were used in the single-letter condition as well, but were presented in lowercase. We chose to present the single characters in lowercase to replicate experimental results from our lab (Wong, Jobard, James, James, \& Gauthier, 2005). The stroke counts of the digit strings were equated with those of the letter strings as much as possible and included the digits $2-9$. We equated the visual complexity of the Chinese characters with that of the Roman letters as much as possible by using only simple characters (maximum four strokes). For each string, we used seven of a total of eight Chinese characters.

The single-character and string stimuli were presented in separate runs. Each stimulus was presented for $825 \mathrm{msec}$, followed by a 175 -msec pause. There were 16 stimulus presentations per block, resulting in 16-sec blocks. Each block was followed by a 10-sec fixation cross. There were 3 blocks for each stimulus type, resulting in a total of 9 blocks within a given single-character run and 12 blocks in the character-string runs. Blocks were pseudorandomized within each run, and trials were randomized within each block. Each single-character run was $4 \mathrm{~min}$ long, and each string run was $5 \mathrm{~min}$ long. Three runs of each stimulus type were presented.

\section{Imaging Parameters and Analysis}

Imaging was performed using a 3-Tesla, whole-body GE MRI system and birdcage head coil located at the Vanderbilt Medical Center. The field of view was $24 \times 24 \times 14 \mathrm{~cm}$, with an in-plane resolution of $64 \times 64$ pixels and 20 contiguous oblique coronal scan planes per volume (whole brain), resulting in a voxel size of $3.75 \times 3.75 \times 7.0 \mathrm{~mm}$. Images were collected using a T2*-weighted EPI acquisition $(\mathrm{TE}=25 \mathrm{msec}, \mathrm{TR}=2,000 \mathrm{msec}$, flip angle $=$ $70^{\circ}$ ) for blood oxygen-level dependent (BOLD)-based imaging (Ogawa et al., 1993). High-resolution T1-weighted anatomical volumes were also acquired using a 3-D fast spoiled grass acquisition $\left(\mathrm{TI}=400 \mathrm{msec}, \mathrm{TE}=4.18 \mathrm{msec}, \mathrm{TR}=10 \mathrm{msec}\right.$, flip angle $\left.=20^{\circ}\right)$.

The functional data were analyzed using the Brain Voyager (www .brainvoyager.com) multistudy general linear model (GLM) procedure and in-house programs written in MATLAB (www.mathworks .com). A GLM analysis allows for the correlation of predictor variables or functions with the recorded activation data (criterion variables) across scanning sessions. The predictor functions are based on the blocked stimulus presentation paradigm of the particular run being analyzed and represent an estimate of the predicted hemodynamic response during that run. To properly model the hemodynamic response, the predictors are represented as the stimulus protocol boxcar functions convolved with the appropriate gamma function $(\Delta=2.5, \tau=1.25)$ estimate of a typical hemodynamic response (Boynton, Engel, Glover, \& Heeger, 1996). Group activation was considered to be above threshold if it met the following criteria in our random effects analysis: (1) significant at $p<.001$, uncorrected with a cluster threshold of 10 contiguous $3-\mathrm{mm}$ isometric voxels, and (2) peak activity within a cluster at $p<.0001$, 
uncorrected. Note that the maps are thresholded at $p<.001$. For individual analyses, activation was considered significant at $p<.006$, corrected using the false discovery rate (FDR) method, which controls for the expected proportion of false positive voxels among those that are suprathreshold (Genovese, Lazar, \& Nichols, 2002). A cluster threshold of 10 contiguous isometric $3-\mathrm{mm}$ voxels was also applied.

\section{RESULTS}

\section{Behavioral Results}

The subjects performed a 1-back matching task on single characters and on strings of characters (see the Method section for a more detailed description). The matching task was designed to be quite easy, and for this reason we did not expect differences in performance between conditions. Because accuracy of performance in letter recognition tasks has been shown to affect signal strength in fMRI (Garrett et al., 2000), it was important to ensure low variance in response accuracy among subjects and across tasks. Performance was at ceiling in all tasks except the 1-back matching task with Chinese character strings. The subjects tended to make more false alarms in this condition, but the difference in performance in comparison with that in all other conditions was not significant $[F(3,7)=1.65]$. Additional behav- ioral results from 18 subjects tested outside of the scanner, with a response required for each stimulus rather than as in a go-no-go task, also showed that performance on a 1-back task with Chinese characters was poorer than performance on the same type of task with Roman letters or digits (Figure 2; see Table 1). A paired $t$ test revealed no significant difference between letter strings and pseudowords; therefore, the behavioral data for these two stimuli were combined in further analyses. A stimulus category (Roman vs. Chinese vs. digits) $\times$ stimulus length (isolated character vs. character strings) ANOVA yielded significant effects of stimulus category on accuracy $[F(2,34)=8.02, p=.001]$ and reaction time $[F(2,34)=$ $11.58, p<.001]$, the Chinese character conditions leading to significantly lower accuracies and longer reaction times than did the Roman letter and digit conditions. (A post hoc Bonferroni test for accuracy yielded $p<.01$ for Roman letters and $p<.05$ for digits; for reaction times, it yielded $p<.01$ for Roman letters and $p<.01$ for digits.) Although the effect of stimulus length on accuracy failed to reach significance $[F(1,17)=2.07, p=.17]$, the subjects required more time to complete the 1-back task on strings than on single characters $[F(1,17)=59.21, p<$ $.001]$. This was the case even though the subjects needed to attend only to a single, clearly marked letter in the string
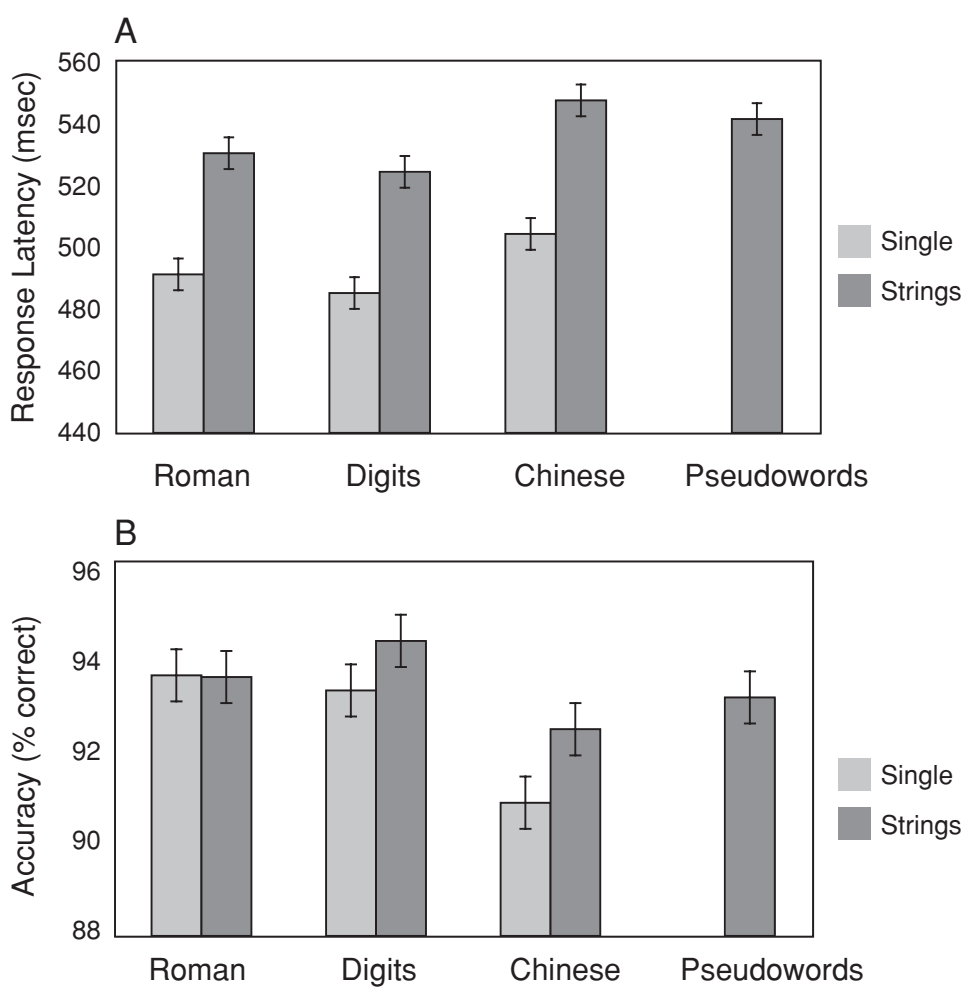

Figure 2. Behavioral results from the 1-back task outside the scanner with a target stimulus (Roman letters, digits, Chinese characters, and pseudowords) presented either in isolation or in strings. Error bars represent pooled standard errors. Performance at matching $\mathrm{Chinese}$ characters was less accurate and took longer than performance with letters and digits. Letters presented in isolation led to faster responses than did strings, independent of stimulus category. 
Table 1

Mean Response Times (in Milliseconds, With Standard Errors) and Mean Accuracy (in Percentages, With Standard Errors) for the 1-Back Task Outside the Scanner With Target Stimuli (Roman Letters, Chinese Characters, and Digits) Presented in Isolation and in Strings

\begin{tabular}{|c|c|c|c|c|c|c|c|c|}
\hline \multirow[b]{3}{*}{ Target Stimuli } & \multicolumn{8}{|c|}{ Presentation } \\
\hline & \multicolumn{4}{|c|}{ Single Letters } & \multicolumn{4}{|c|}{ Strings } \\
\hline & RT & $S E$ & Acc. & $S E$ & $\mathrm{RT}$ & $S E$ & Acc. & $S E$ \\
\hline Letters & 491 & 16 & 93.56 & 1.03 & 530 & 18 & 93.52 & 0.87 \\
\hline Digits & 485 & 17 & 93.23 & 1.04 & 524 & 17 & 94.29 & 0.88 \\
\hline Chinese characters & 504 & 18 & 90.84 & 1.55 & 547 & 19 & 92.41 & 1.18 \\
\hline Pseudowords & & & & & 541 & 18 & 93.08 & 1.07 \\
\hline
\end{tabular}

Note-Acc., accuracy.

task. However, the lack of an interaction between stimulus type and length for the accuracy scores $[F(2,34)=$ $2.02, p=.15]$ or reaction times $[F(2,34)=0.20, p=$ $.82]$ indicates that the increase in difficulty for strings was equivalent for each condition.

\section{Imaging Results}

The imaging data were analyzed using the Brain Voyager multistudy GLM procedure. Statistical parametric maps (SPMs) were calculated in a group analysis of all 8 subjects with a random-effects model.

\section{Group Analyses}

SPMs. We first localized the standard VWFA using the contrast of pseudowords to consonant strings (Dehaene et al., 2002). This area was found in its typical location [peak $t(7)=6.08, p<.0001$, uncorrected; TCs $-46,-54,-7$; see Figure $3 \mathrm{~A}]$.

To find brain regions involved in processing single characters, we contrasted neural activation for Roman letters with that obtained for digits and for Chinese characters (letters-digits and letters-Chinese characters; Figure $3 \mathrm{~B}$ ). The only activation in the occipitotemporal cortex that resulted from this contrast was in the left anterior fusiform gyrus [peak $t(7)=7.06, p<.0001$; TCs $-42,-37,-3]$. Two other regions were active: an area in the inferior parietal lobe (TCs $-40,-21,56)$ and an area in the ventral prefrontal cortex (TCs $-50,1,17)$. Both of these regions have been implicated in the phonetic and semantic processes associated with word perception (for a review, see Jobard et al., 2003). Because these are not usually associated with visual processing, a further analysis of these areas was not warranted here.

Similarly, we contrasted the BOLD response for Roman letter strings with that for digit strings and Chinese character strings (Figure 3C). We found one area of significant activation in the ventral occipitotemporal cortex, again on the left fusiform gyrus but posterior to the area that showed specialization for single-letter processing [peak $t(7)=6.19, p<.0001$; TCs $-31,-64,-5$ ]

Additional information concerning the degree of specialization within these three areas was obtained by plotting the subjects' responses in the conditions that were not used to define them (see the graphs in Figure 3). In the VWFA, we observed no difference between letter strings and digit strings $[t(7)=0.83]$ and a trend toward a difference between single letters and digits $[t(7)=$ 2.97, $p<.05$, Bonferroni corrected], but no difference between letters and Chinese characters $[t(7)=0.56]$. In the region defined by its selectivity for single Roman characters, we found no significant difference among our string conditions (Figure 3B). When we compared the activation to single characters within the area defined by its selectivity for Roman letter strings, we found no difference between letters and digits $[t(7)=2.01$, n.s. with correction] but greater activation to letters than to Chinese characters $[t(7)=5.29, p<.001$, Bonferroni corrected; see Figure 3C and Table 2 for descriptive statistics]. Possible explanations for this may be the stronger familiarity of letters and digits, and the greater difficulty of the task with Chinese characters.

These group analyses are especially useful in comparing our results with those of prior studies in which data was analyzed in the same manner (e.g., Cohen et al., 2000; Dehaene et al., 2002). Specifically, not being localized within the same study, the VWFA and the area that we find is selective for letter strings may be mistaken for the same region because they are relatively close. Our ability to localize the VWFA is important because it shows that this area can be engaged in a task that required selective attention to only one letter of the string, and it also allows for a spatial reference to compare with our other findings.

The finding of two areas that responded preferentially to either single Roman letters or strings of Roman letters, in comparison with digit and Chinese character controls, suggests specialization that cannot be explained by simple shape differences such as those between letter strings and complex stimuli (e.g., faces or textures used in other studies; see Hasson et al., 2002; Puce et al., 1996).

We do not believe that the different activation patterns that we see between single-letter stimuli and strings are due to case differences in the two types of stimuli. For this to be true, one would have to posit that uppercase single letters would not activate the same region as efficiently as the lowercase letters that we used. There is no reason to believe that this would be the case, given that we have localized the same anterior fusiform area in two other experiments in which we used uppercase letters (James \& Gauthier, 2005; James et al., 2004). Also, several studies (e.g., Dehaene et al., 2004; Dehaene et al., 2001; Polk \& Farah, 2002) have been conducted to investigate case invariance using fMRI and converge to suggest that the representation in the left fusiform gyrus is invariant to case. This is not merely a null finding, because Dehaene et al. (2002) identified areas in right extrastriate cortex (and, marginally significant, in the corresponding area on the left) that showed case-dependent priming. Thus, the literature suggests that if there is a case effect, it should be found mainly in the right hemisphere and posterior to the VWFA. In contrast, our strongest interaction between single letters and strings is found in an area that is clearly anterior to the VWFA. In- 

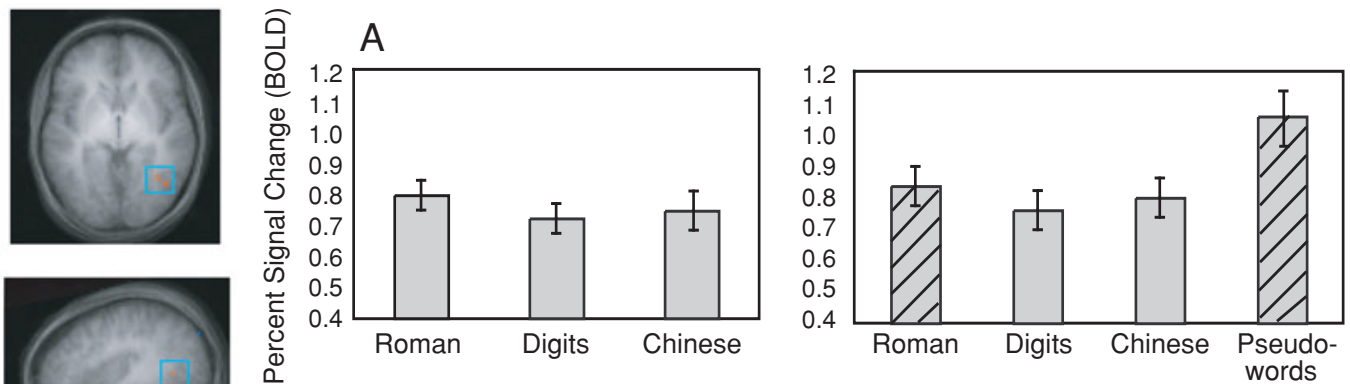

Pseudowords - letter strings: peak $t(7)=6.80, p<.0001$. TC: $-46,-54,-7$ (VWFA overlap).
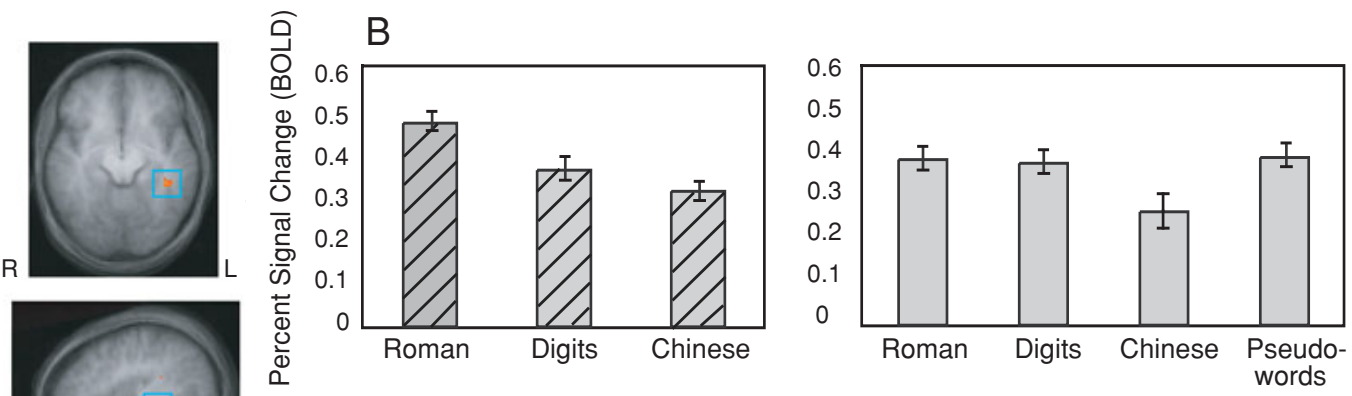

Single Roman letter area: peak $t(7)=7.06, p<.0001$. TC: $-42,-37,-3$.
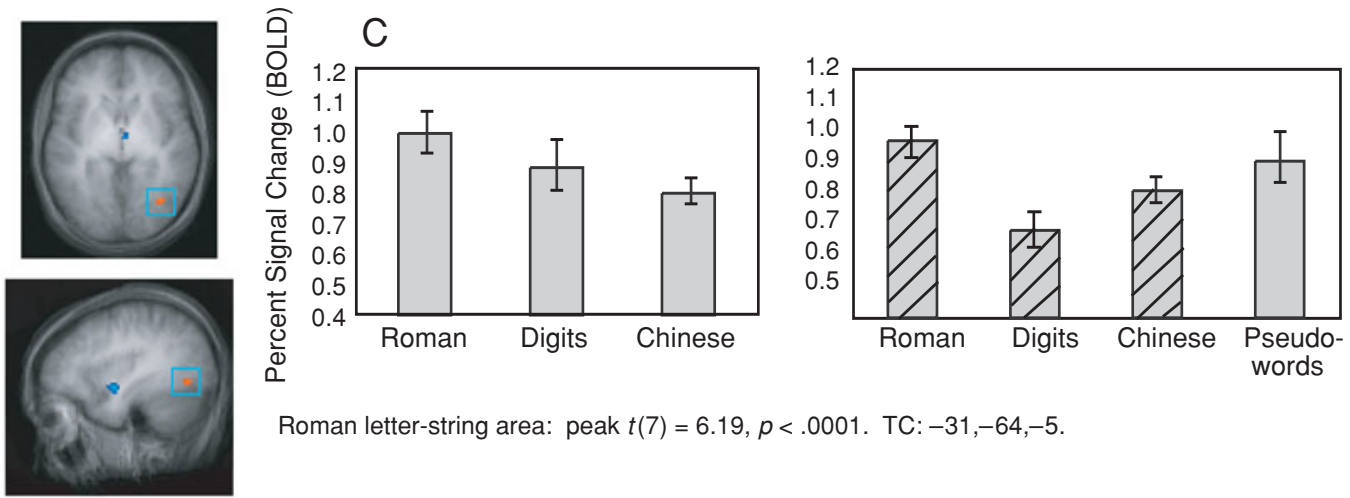

Roman letter-string area: peak $t(7)=6.19, p<.0001$. TC: $-31,-64,-5$.

Figure 3. Statistical parametric maps (SPMs) of averaged activation for 8 subjects. (A) The contrast pseudowords minus letter strings $[t(7)=4.80, p<.001$; peak $t(7)=6.80, p<.0001]$. Peak activation was $-46,-54,-7$. The more lateral region peaked at $-55,-60,-7$. Both regions were relatively small, but they passed a contiguity filter of ten 3-mm isometric voxels. In the medial region, activation ranged as follows: $(x)-45$ to $-47,(y)-52$ to -56 , and $(z)-6$ to -9 ; in the lateral area, ranges of activation were $(x)-54$ to $-58,(y)-58$ to -62 , and $(z)-6$ to -9. (B) The conjunctions of single Roman characters versus Chinese characters and of Roman characters versus digits $[t(7)=4.80, p<.001$; peak $t(7)=7.06, p<.0001]$. The Talairach coordinates for peak activation were $-42,-37,-3$. The activation ranged from $(x)-39$ to $-45,(y)-32$ to -40 , and $(z) 4$ to -6 . (C) The conjunctions of Roman letter strings versus Chinese character strings and letter strings versus digit strings $[t(7)=4.80, p<$ .001 ; peak $t(7)=6.19, p<.0001$. Peak activation for $(C)$ was $-31,-64,-5$; activation ranged from $(x)-29$ to $-34,(y)-59$ to -70 , and $(z) 0$ to -7 . Graphs represent percent signal change for each condition within the areas of high activation shown in the SPMs. Bars with diagonal lines represent the conditions used to produce the SPMs. Error bars represent standard error of the between-subjects mean for each condition; therefore, they do not reflect significance. Means and standard errors for all conditions are reported in Table 2.

deed, there is quite a bit of convergence, suggesting that representations become progressively more abstract as one moves anteriorly in the left fusiform gyrus.
Moreover, it is interesting that the single-letter area did not show specialization for letter strings even though the strings were composed of the same characters that 
Table 2

Means (and Standard Errors) for Each Condition in the SPM Group Analysis and the ROI Analysis

\begin{tabular}{|c|c|c|c|c|c|c|c|c|c|c|c|c|c|c|}
\hline & \multicolumn{2}{|c|}{$\begin{array}{l}\text { Roman } \\
\text { Letters }\end{array}$} & \multicolumn{2}{|c|}{ Digits } & \multicolumn{2}{|c|}{$\begin{array}{c}\text { Chinese } \\
\text { Characters } \\
\end{array}$} & \multicolumn{2}{|c|}{$\begin{array}{l}\text { Roman } \\
\text { Strings }\end{array}$} & \multicolumn{2}{|c|}{$\begin{array}{c}\text { Digit } \\
\text { Strings }\end{array}$} & \multicolumn{2}{|c|}{$\begin{array}{c}\text { Character } \\
\text { Strings }\end{array}$} & \multicolumn{2}{|c|}{ Pseudowords } \\
\hline & $M$ & $S E$ & $M$ & $S E$ & $M$ & $S E$ & $M$ & $S E$ & $M$ & $S E$ & $M$ & $S E$ & $M$ & $S E$ \\
\hline Figure 3: PW area & 0.81 & 0.08 & 0.73 & 0.09 & 0.76 & 0.12 & 0.89 & 0.09 & 0.80 & 0.07 & 0.85 & 0.09 & 1.13 & 0.19 \\
\hline Figure 3: letter area & 0.24 & 0.04 & 0.19 & 0.06 & 0.16 & 0.04 & 0.16 & 0.05 & 0.19 & 0.04 & 0.15 & 0.07 & 0.17 & 0.06 \\
\hline Figure 3: string area & 1.08 & 0.15 & 1.13 & 0.18 & 0.95 & 0.12 & 1.40 & 0.09 & 1.29 & 0.16 & 1.27 & 0.12 & 1.33 & 0.19 \\
\hline Figure 4: $y=-35$ & 0.25 & 0.02 & 0.14 & 0.04 & 0.13 & 0.04 & 0.10 & 0.05 & 0.15 & 0.04 & 0.14 & 0.07 & 0.14 & 0.06 \\
\hline Figure 4: $y=-45$ & 0.43 & 0.07 & 0.36 & 0.09 & 0.29 & 0.08 & 0.29 & 0.10 & 0.31 & 0.07 & 0.32 & 0.13 & 0.38 & 0.14 \\
\hline Figure 4: $y=-55$ & 0.81 & 0.18 & 0.76 & 0.19 & 0.62 & 0.15 & 0.61 & 0.13 & 0.60 & 0.11 & 0.66 & 0.14 & 0.70 & 0.20 \\
\hline Figure 4: $y=-65$ & 1.13 & 0.19 & 1.09 & 0.19 & 0.94 & 0.13 & 1.07 & 0.16 & 1.10 & 0.12 & 1.19 & 0.10 & 1.16 & 0.20 \\
\hline
\end{tabular}

Note-PW, pseudoword.

were presented in isolation. One possibility is that we did not replicate selectivity in this region because of regression to the mean. That is, if there is no true specialization for Roman letter stimuli, it may be possible to find statistically significant voxels by accident, but this would be unlikely to be replicated in a second condition (thereby artificially suggesting a double dissociation). This appears unlikely because in both cases the selectivity was found in the left fusiform gyrus, as could be expected from the literature. Nonetheless, to address this possibility further, we compared the responses to all of our conditions in more neutral regions of interest, defined on the basis of coordinates reported in prior studies.

ROI analysis. To define the ROIs, we calculated the range of individual subject activation in Polk et al. (2002). We chose to select our ROIs on the basis of Polk's results for several reasons: First, they used digit strings as a control condition and found specialization for letter strings over digit strings. Second, we modeled our string stimuli after theirs, using the same digits and letters. Third, in one of their experiments, they used a 1-back matching task similar to ours. Here, we ask whether any part of the left fusiform gyrus is specialized for letters or letter strings as opposed to unfamiliar nonletter shapes of comparable complexity (e.g., Chinese characters).

After calculating the limits of a large ROI on the basis of the range of the subjects' activation in Polk et al.'s (2002) experiments, we split the region into four smaller, equalsized ROIs of $10 \times 10 \mathrm{~mm}^{3}$, each along the posterioranterior axis (see Figure 4). We also replicated these ROIs in the right hemisphere for comparison. We split this region along the posterior-anterior axis because previous studies have shown differences in response properties of anterior versus middle versus posterior regions of the left fusiform gyrus (see Dehaene et al., 2004). We then calculated percent signal change for each condition relative to fixation baseline and first performed an ANOVA on all conditions in each hemisphere (for means and standard errors, see Table 2). A 2 (length: single vs. strings) $\times$ 4 (ROI) $\times 3$ (stimulus: letters vs. digits vs. Chinese characters) ANOVA was first conducted in the right hemisphere, where most prior studies failed to find much selectivity for letter strings. An effect of ROI surfaced $[F(3,18)=18.65, p<.0001]$ : Activation increased as
ROI was more posterior, and this effect was also seen in the left hemisphere (below). In addition, there was an ROI $\times$ length $\times$ character interaction $[F(3,36)=2.4$, $p<.05]$, which was due to differences in activation to Chinese characters. In the most posterior ROI, single Chinese characters elicited less activation than did letters and digits, whereas Chinese strings in this region resulted in higher activation. However, neither of these simple effects reached significance. In the left hemisphere, an ANOVA revealed a significant length $\times$ character interaction $[F(2,12)=4.05, p<.05]$. In addition, the main effect of ROI was significant $[F(3,18)=29.01$, $p<.0001]$ : The more posterior the ROI, the greater was the overall activation (see Figure 4). We followed up with two more ANOVAs in the left hemisphere, one for single characters $(4 \times 3)$ and one for strings $(4 \times 4)$, in order to include the pseudowords condition as a variable. The ANOVA on single characters showed main effects of both ROI $[F(3,18)=19.99, p<.0001]$ and character $[F(2,12)=4.3, p<.03]$. As ROI became more posterior, activation increased (see Figure 4), but, more importantly, simple effects revealed that significantly more activation occurred for letters than for Chinese characters overall $[t(31)=4.57, p<.001]$ and more activation occurred for letters than for digits overall $[t(31)=3.30$, $p<.01]$. The ANOVA on strings led to a main effect of ROI only $[F(3,18)=36.89, p<.0001]$. Again, percent signal change increased as the ROI position moved posterior (see Figure 4). This analysis demonstrates an interesting difference between selectivity for single letters and selectivity for digits when presented alone or in strings. A very large expanse of cortex in the left fusiform gyrus responds more to letters and digits than to Chinese characters when they are presented alone, but not when they are shown in strings. This suggests a particularly distributed representation of letters (Ishai, Ungerleider, Martin, Schouten, \& Haxby, 1999) as well as higher order processing of consonant strings, which leads them to be processed as "more than letters." The ROI analysis is also consistent with the SPM analysis in terms of the selectivity for single letters over both digits and Chinese characters in anterior fusiform gyrus. However, although the VWFA and the area found to be selective for strings overlap to some extent with some of the four ROIs, se- 


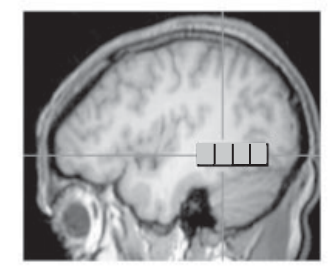

$$
\begin{aligned}
& X=-40 \\
& Y=(-35,-45,-55,-65) \\
& Z=-5
\end{aligned}
$$
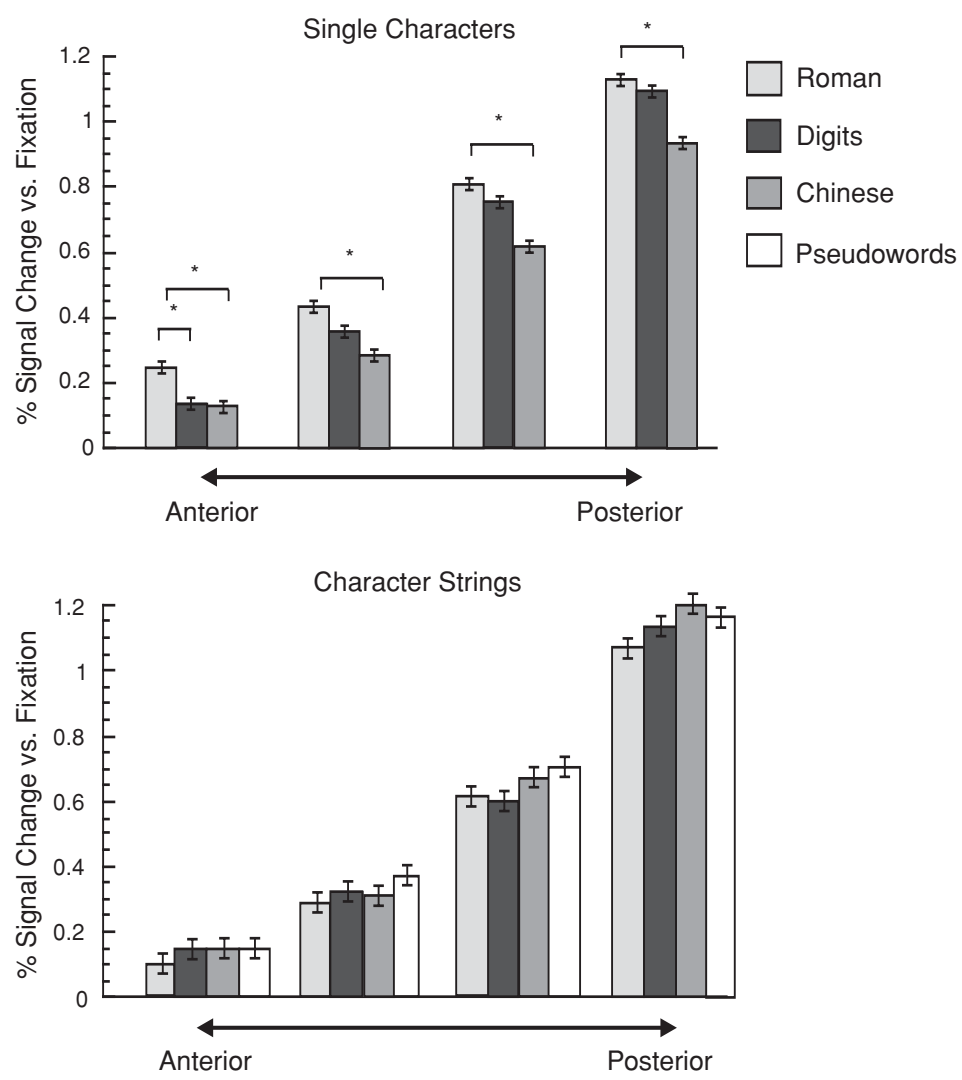

Figure 4. Results of the left hemisphere ROI analysis. Boxes superimposed on the brain image are $10 \times 10 \mathrm{~mm}^{3}$ ROIs that were selected on the basis of results reported in Polk et al. (2002). Graphs represent percent signal change in these ROIs for all conditions tested. The $x$-axis represents the four ROIs as one moves from anterior $(y=-35)$ cortex to posterior $(y=-65)$ cortex. The top graph shows activation to single characters, whereas the bottom graph represents activation to string stimuli. Asterisks indicate significant simple effects $(p<.05)$. Error bars represent mean square error terms for the multisubject general linear models carried out for both single characters $\left(M S_{\mathrm{e}}=.05\right)$ and strings $\left(M S_{\mathrm{e}}=\mathbf{. 0 8 5}\right)$. Means and standard errors are reported in Table 2.

lectivity at the string level was not observed. This suggests a less distributed pattern of activity for strings, in which small foci of selectivity may be overpowered by nonselective voxels in these large ROIs. This is also consistent with prior work showing considerable individual differences in the location of small foci of activity for letter strings (Polk et al., 2002). In that study, the letterstring areas found in individual subjects did not overlap sufficiently across individuals to emerge in a group composite. To further explore this issue, we conducted individual analyses similar to those performed by Polk et al. to investigate the pattern of functional specialization for strings and single letters in individual subjects.

\section{Individual Analyses}

Polk et al. (2002) suggested that the large individual variability in the spatial location of letter-string-selective areas was due to individual differences in neural development, because processing letters is not an innate skill. However, we found regions within the ventral processing stream that responded more to single Roman characters than to control stimuli, even though these are unlikely to engage an innate skill. Another explanation may reside in different strategies used by different subjects when processing strings. For example, some may scan the whole string whereas others may fixate on the center; some may relate the string to words they know, and others may 
not. In contrast, there may be fewer processing strategies available for single characters.

Figure 5 presents individual maps for the 5 (out of 8) subjects who showed significant activation to Roman letter strings relative to digit strings. These regions all fell within the left fusiform gyrus, and, as was the case with Polk et al.'s (2002) results, there was considerable variability among subjects in the location of the small foci of activation (see Table 3A for TCs).

Not only did letter strings elicit more activity than digit strings $[t(4)=3.97, p<.01$, Bonferroni corrected] and Chinese character strings $[t(4)=4.29, p<.01]$, but single letters also led to a (Bonferroni-corrected) trend toward more activity than that shown for single Chinese characters $[t(4)=2.16, p<.04]$ but not more than that shown for digits $[t(4)=1.18$, n.s. $]$. In addition, there was no difference between activation to pseudowords and activation to letter strings $[t(4)=0.38$, n.s.].

We also compared single letters with single digits in individuals, and in 6 subjects we found an active area that was generally more anterior and medial to the letter string area described above (Figure 5B, Table 3B). As in our group analyses, single letters activated this region more than did digits $[t(5)=3.7, p<.01]$ and Chinese characters $[t(5)=4.0, p<.01]$, whereas string stimuli did not show differential activation.

These findings support and considerably extend those obtained by Polk and his colleagues (Polk \& Farah, 1998, 2002; Polk et al., 2002). First, we replicated the finding of a small area within a large region of the left fusiform gyrus that is more specialized for letter-string processing than for digit-string processing. Second, we showed that this specialization holds in a 1-back task when subjects focused on the central character of a string, as well as in the passive viewing task and full-string matching task used in the prior studies. Third, in addition to selectivity for letter strings over digit strings, we found evidence in the left fusiform gyrus of some individuals for selectivity for single letters over single digits. In each case, Chinese characters (or Chinese strings) were found to elicit no more activity in these areas than did digits in the baseline condition. Fourth, and in alignment with our other analyses, we found a certain degree of dissociation between single characters and strings. In each case, areas defined as selective for letters as opposed to digits (or letter strings as opposed to digit strings) failed to show the equivalent selectivity in the other condition.

\section{DISCUSSION}

An oft-cited misconception is that, in terms of neural activation, letters may not be an effective stimulus in comparison with words (Price \& Devlin, 2003). This conclusion is based on the fact that consonant strings do not activate the VWFA to the same extent as words do (Cohen et al., 2000; Dehaene et al., 2002). Here, we show that individual letters are an effective stimulus for activating the ventral occipital and temporal cortices. In- spection of Figure 4 reveals how single characters elicit at least as much activity as character strings with reference to a fixation baseline, throughout a large expanse of left fusiform territory. This class of stimuli was overlooked in past research, perhaps due to the obvious relevance of letter strings to the study of reading. Neural specialization for processing letters has been demonstrated here in three ways: First, the use of group SPMs revealed the presence of a brain area that responded more to single letters than to other, visually similar stimuli, as well as that of a more posterior area that responded more to letter strings than to other string stimuli. Second, using an ROI approach, we showed that a vast expanse of the left fusiform gyrus responded more to single letters than to other single characters and character strings. Third, we replicated our group results in individuals by showing that areas in individuals that appear specialized to process letter strings rather than digit strings do not show a preference for single letters over single digits. Conversely, dissociable areas that were selective for single letters over single digits showed no preference for letter strings over other string types. A recent eventrelated potential study also demonstrated that an early waveform (N170) is of greater magnitude for single letters of a familiar writing system, whether Roman or Chinese, than for other, similar characters, such as false fonts or Chinese characters in non-Chinese readers (Wong, Gauthier, Woroch, DeBuse, \& Curran, 2005). Together, these findings stress the importance of considering single letters in future studies of the neural substrate for printed text.

\section{Single Letters Versus Letter Strings}

We found that perceiving single letters engages a large portion of the ventral visual cortex, but that most of this area is not activated more by strings of letters than other string types. Because strings are simply a group of letters, this result is somewhat counterintuitive. However, two of our three analyses also indicate that a more posterior region responded more to letter strings than to other string types. Because we found string-sensitive regions in our SPM analyses but not in our ROI analyses, it seems that the string-selective areas are just very small. The activation to strings was overshadowed in our ROI analyses, leading to reduced sensitivity to letter strings in comparison with other string types. Given that there are only a few studies in which single characters were used as stimuli, we can only speculate as to why letters and letter strings activate different regions of the visual cortex. Perhaps letter strings are processed more like words than like groups of letters (Jobard et al., 2003). The idea that letter strings are processed similarly to words is supported by our finding that the area specialized for letter strings was located near the VWFA. In contrast, the area most sensitive to isolated letters was significantly more anterior to the VWFA. Our results nonetheless confirm that letter strings are not processed in the same way as pseudowords are, as reflected in the 


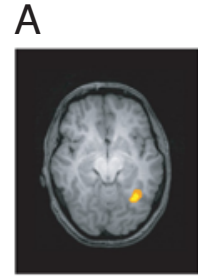

$\mathrm{S} 1(z=-6)$

$t=3.32, q<.006$

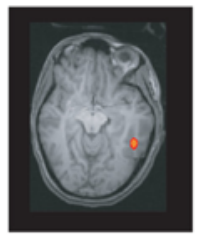

$\mathrm{S} 2(z=-9)$

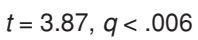

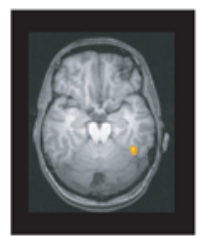

$\mathrm{S} 3(z=-12)$

$t=4.16, q<.006$

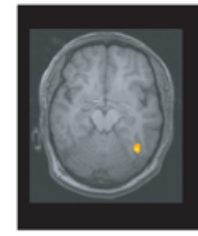

$\mathrm{S} 4(z=-7)$ $t=3.77, q<.006$

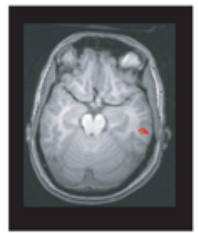

$\mathrm{S} 5(z=-10)$

$t=3.62, q<.006$
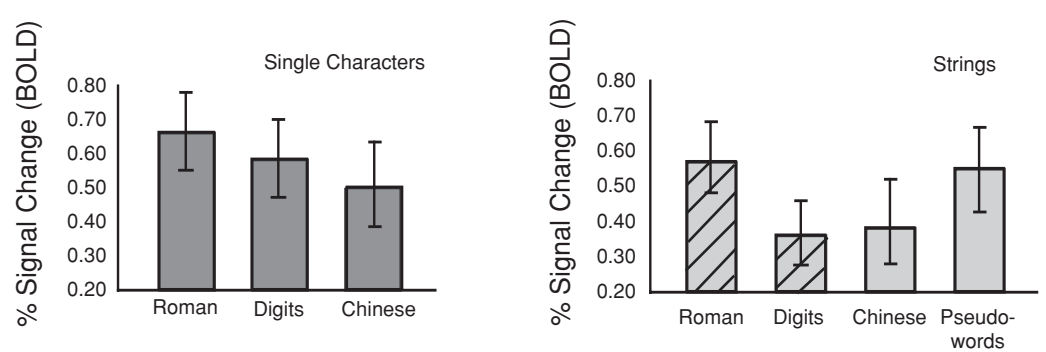

$\mathrm{B}$

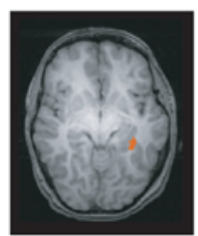

$\mathrm{S} 1(z=-6)$

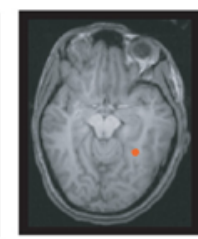

$\mathrm{S} 2(z=-9)$

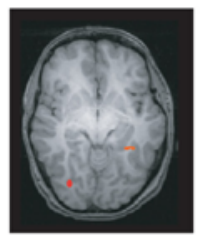

$\mathrm{S} 3(z=-5)$

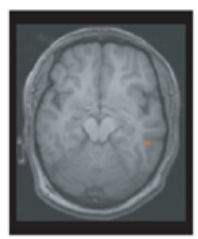

$\mathrm{S} 4(z=-7)$

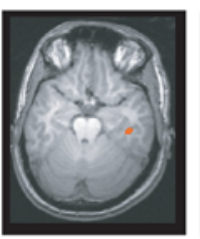

$\mathrm{S} 5(z=11)$

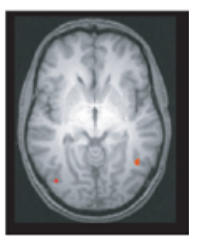

$\mathrm{S} 6(z=-5)$ $t=3.68, q<.006 t=3.19, q<.006 \quad t=3.75, q<.006 t=3.62, q<.006 \quad t=3.39, q<.006 t=3.87, q<.006$
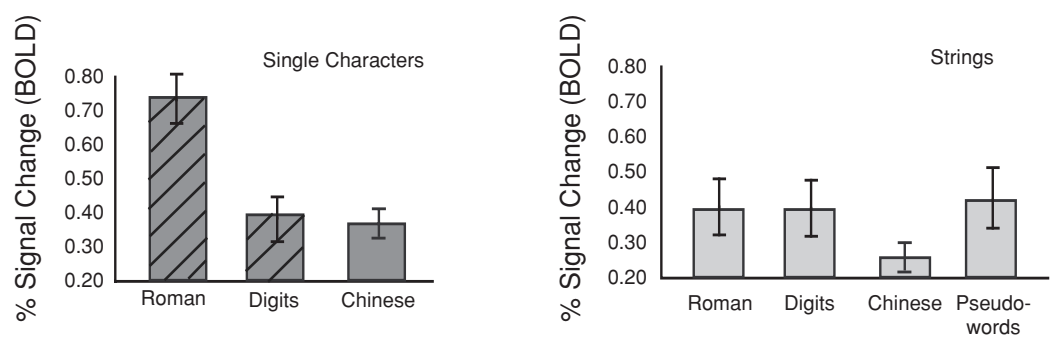

Figure 5. Statistical parametric maps (SPMs) depicting (A) the contrast between letter strings and digit strings in 5 individual subjects and $(B)$ the contrast between single letters and digits. Activations are thresholded on the basis of fusiform activation of a contiguous cluster size greater than 10 isometric 3-mm voxels and a $q$ value of less than $\mathbf{. 0 5}$. All are corrected for multiple comparisons using the false discovery rate (FDR) method. The FDR method used here on the individual subject data uses a $q$ value rather than a $p$ value. Graphs represent the percent signal change of the given contrast in the activated region averaged across individuals. Bars with diagonal lines represent the conditions used to produce the SPMs. Error bars represent standard error of the mean within each condition. Descriptive statistics are presented in Table 3.

activity of the VWFA. Pseudowords may be influenced by phonological processing, whereas letter strings, although visually similar to words, do not benefit from such processing. Our results add to the puzzle of the VWFA by showing, on one hand, heightened activation of this area in response to pseudowords in comparison with letter strings, and on the other hand, no specialization for letter strings over digit strings or Chinese characters, even though roughly the same area $(y=-55$ in our ROI analysis) responded more to single Roman letters than to single digits and single Chinese characters.
These puzzles regarding the processes invoked by letter strings in the left fusiform cortex are not unique to our study. They are consistent with current controversies regarding the role of the VWFA and why this area appears to be recruited in many tasks that should not involve the processing of visual word forms (Cohen \& Dehaene, 2004; Price \& Devlin, 2003). In contrast, the pattern of response to single characters in the left fusiform gyrus is one that seems much more intuitive than the pattern of response to strings. That is, our subjects had more experience with letters than with digits, and more expe- 
Table 3A

Talairach Coordinates for Individual Activations in the Subtraction of Digit Strings From Letter Strings

\begin{tabular}{ccccc}
\hline & \multirow{3}{c}{$\begin{array}{c}\text { Peak } \\
\text { Coordinates } \\
\text { Subject }\end{array}$} & \multicolumn{3}{c}{ Range } \\
\cline { 3 - 5 } & \multicolumn{1}{c}{ S,y,z) } & $x$ & $y$ & $z$ \\
\hline 1 & $-33,-52,-6$ & $-27,-42$ & $-42,-59$ & $0,-12$ \\
2 & $-50,-44,-9$ & $-43,-51$ & $-40,-49$ & $-2,-11$ \\
3 & $-41,-50,-12$ & $-38,-44$ & $-42,-53$ & $-9,-15$ \\
4 & $-35,-53,-7$ & $-32,-42$ & $-44,-63$ & $-5,-9$ \\
5 & $-55,-29,-10$ & $-30,-64$ & $-27,-35$ & $-8,-19$ \\
\hline
\end{tabular}

Note-Numbers in bold indicate ranges that overlap with the VWFA (Cohen et al., 2000).

Table 3B

Talairach Coordinates for Individual Activations in the Subtraction of Single Digits From Letters

\begin{tabular}{|c|c|c|c|c|}
\hline \multirow[b]{2}{*}{ Subject } & \multirow{2}{*}{$\begin{array}{c}\text { Peak } \\
\text { Coordinates } \\
(x, y, z)\end{array}$} & \multicolumn{3}{|c|}{ Range } \\
\hline & & $x$ & $y$ & $z$ \\
\hline 1 & $-33,-40,-6$ & $-27,-35$ & $-35,-44$ & $-2,-9$ \\
\hline 2 & $-35,-46,-9$ & $-33,-36$ & $-44,-47$ & $-1,-12$ \\
\hline 3 & $-37,-42,-5$ & $-34,-38$ & $-41,-44$ & $-3,-7$ \\
\hline 4 & $-43,-45,-7$ & $-42,-44$ & $-44,-46$ & $-4,-10$ \\
\hline 5 & $-45,-29,-7$ & $-43,-47$ & $-27,-32$ & $-5,-13$ \\
\hline 6 & $-43,-47,-5$ & $-41,-44$ & $-44,-49$ & $-3,-6$ \\
\hline
\end{tabular}

Note-Numbers in bold indicate ranges that overlap with the VWFA (Cohen et al., 2000. Note that no subject overlaps in all three dimensions.

rience with digits than with Chinese characters, and the fusiform activity reflects this pattern. These results suggest that the use of single characters may be better suited for the study of early visual processing of letters in this region. The complex pattern of response, including both comparisons with strings and comparisons with single characters, is compatible with the idea that the left fusiform gyrus is heterogeneous and may include both neurons influenced by perceptual experience and others involved in more abstract visual word form perception (McCandliss et al., 2003; Polk \& Farah, 2002). The response to single characters could reflect mainly the former, whereas the puzzling pattern of response to strings may result from the activity of the two functionally different systems. Neuroimaging methods designed to dissociate spatially overlapping neuronal populations could be used to investigate this hypothesis (Grill-Spector et al., 1999).

\section{Perceptual Expertise for Letters and the Process Map Hypothesis}

Why would the cortex be specialized for processing letters? After all, the ability to recognize letters more efficiently than other, similar items cannot be a genetically predetermined ability. Some researchers postulate that the cortex has regions that specialize in processing certain categories of objects, such as faces (Kanwisher, McDermott, \& Chun, 1997), body parts (Downing, Jiang, Shuman, \& Kanwisher, 2001), and places, such as buildings (Epstein \& Kanwisher, 1998), whereas the remaining ventral temporal cortex generally supports the recognition of all other objects (Grill-Spector, 2003). Whether or not specialization for some of these categories is innate is still a topic of contention for those who believe that object category is an organizing principle of the ventral temporal cortex. Others contend that eccentricity maps in the ventral temporal cortex can account for category specialization (Malach, Levy, \& Hasson, 2002). For example, stimuli that require foveal analyses for the extraction of fine-detailed information, such as faces and letter strings, activate regions of cortex that have a central (foveal) representation. Items that do not require a fine-grained analysis, such as houses, activate regions that have peripheral visual field representations. Interestingly, faces and letter strings do seem to activate similar regions (on the ventral fusiform gyrus), but in opposite hemispheres. However, this account alone would not explain differential activation to digits and Chinese characters in comparison with Roman letters, or why the anterior fusiform gyrus treats digits more like Chinese characters than like Roman letters whereas the posterior fusiform gyrus responds to both Roman letters and digits more than to Chinese characters.

Not mutually exclusive with an eccentricity account, the process map hypothesis proposes that the type of processing required for visual recognition determines which part of the ventral temporal cortex will be recruited for any given category (Gauthier \& Logothetis, 2000). For example, to recognize a face we must attend to differences among stimuli at a subordinate level. That is, we must distinguish among individual objects with similar parts in a common configuration. Other sets of stimuli that require the same type of processing should activate a similar region of the brain as do faces. Indeed, observers trained to individuate novel objects on the basis of subordinate-level information show the greatest activation in the putative face-selective area of the fusiform gyrus (Gauthier et al., 1999). This model presupposes that there are many gradients of specialization for different dimensions of visual processing (e.g., eccentricity, complexity, local vs. global processing) that intersect in ventral temporal cortex (see also Malach et al., 2002, for a similar claim). It further postulates that experience in consistently recognizing objects of a category will lead to the automatic recruitment for this category of parts of the cortex best suited for the specific computational requirements of the task. Letter recognition may well constitute a different task than the recognition of most other objects. Letters differ from one another on the basis of their structure, and so are generally recognized at what is termed the basic level (Rosch, Mervis, Gray, Johnson, \& Boyes-Braem, 1976). Common objects (e.g., chairs, birds, plants) also differ in their part structures and are recognized at the basic level, in contrast to faces (and some object categories in expert subjects), which are individuated at a more subordinate level. Thus, to recognize a letter, the visual system must code basic-level differences and disregard subordinate-level differences such as orientation, font, size, and the like. An $A$ must be recognized as an $A$ regardless of whether it is written “隹," "A," or “a," and it must also be distinguished from 
a P. In this sense, expert letter recognition differs from expert face recognition. But expert letter perception also differs from the recognition of common objects in many ways: Letters are seen in the context of very regular arrangements that are constant in orientation, size, and font. Perhaps because the visual system can rely on these regularities and because there is great pressure to recognize letters rapidly, the expert reader may not completely ignore this subordinate information. Instead, the visual system appears to "tune" to this information so as to take advantage of the regularity present in printed text in order to facilitate letter recognition. For instance, letters are better recognized in strings that are regular in font (Sanocki et al., 1998). Regularity in the context of subordinate information is much rarer in common object recognition, suggesting that this "font-tuning" may be relatively specific to expertise with letters (Gauthier, Wong, Hayward, \& Cheung, 2004). Thus, there are principled reasons to believe that expertise with letters would recruit different visual areas than either faces or common objects. In addition, the functional specialization that we found for letters in expert readers cannot be explained by familiarity or by the fact that letters have names. Digits, which are also familiar (as confirmed by comparable behavioral performance) and have known names, failed to elicit a level of activity similar to that produced by Roman letters in the anterior left fusiform gyrus. Note that this specialization for single letters also cannot be explained by a phonological account, because this region does not respond more to pronounceable pseudowords than to unpronounceable letter strings.

Our results generally support prior findings that letters are processed differently from digits (Polk et al., 2002) and extend them to show that this holds true even for isolated characters. Although our more posterior ROIs activate just as strongly to digits as to letters, the most anterior fusiform ROI responded more to letters than to digits. This finding is similar to that of Flowers et al. (2004), who found an anterior site that was letter specific and a more posterior area that responded to letters as well as to symbols. In addition, if we subtract letter activation from digit activation, we do not get reliably greater activation to digits anywhere in the brain. The brain's responses to digits and to letters may differ because our experience with letters is quite different from that with digits. For example, letters are combined into graphemes in a principled fashion, whereas every digit combination is equally acceptable. Some have argued that letters and digits are spatially and temporally clustered in our experience, so that we almost always see letters among other letters, whereas digits are seen in many contexts (e.g., alone, with letters, and with other digits; Polk \& Farah, 1995). Correlation-based learning in a neural network predicts that the two categories would be dissociated (Polk \& Farah, 1998). Other differences between letters and digits include the fact that letters as a group often become a word, invoking extensive seman- tic and phonemic processing, and that there are only nine Arabic digits whereas there are 26 Roman letters. Most of us encounter letters with greater frequency than digits. Although on the surface one would expect the visual processing of letters and that of digits to be very similar, further consideration reveals different processing demands for these two types of stimuli.

In conclusion, our interest in the neural substrates underlying perceptual expertise led us to investigate how the brain responds to visually presented letters. By contrasting fMRI activation to letters with that to digits and Chinese characters, we determined that a large portion of the left fusiform gyrus responded preferentially to Roman letters over other types of characters, whereas a smaller, more focal area in the posterior fusiform gyrus responded more to letter strings than to other character strings. Reading experience leads to a complex pattern of functional specialization in the left fusiform gyrus. Its understanding will necessitate more work involving single letters, as well as the integration of research in visual processing with research in the study of reading.

\section{REFERENCES}

BARON, J., \& ThURSTONE, I. (1973). An analysis of the word-superiority effect. Cognitive Psychology, 4, 207-228.

Black, S. E., \& Behrmann, M. (1994). Localization in alexia. In A. Kertesz (Ed.), Localization and neuroimaging in neuropsychology (pp. 331-376). New York: Academic Press.

BoOKHeIMER, S. (2002). Functional MRI of language: New approaches to understanding the cortical organization of semantic processing. Annual Review of Neuroscience, 25, 151-188.

Booth, J. R., Burman, D. D., Meyer, J. R., Gitelman, D. R., ParRish, T. B., \& Mesulam, M. M. (2002). Modality independence of word comprehension. Human Brain Mapping, 16, 251-261.

Boynton, G. M., Engel, S. A., Glover, G. H., \& Heeger, D. J. (1996).

Linear systems analysis of functional magnetic resonance imaging in human V1. Journal of Neuroscience, 16, 4207-4221.

Cattell, J. M. (1886). The time taken up by cerebral operations. Mind, 11, 220-242.

Cohen, L., \& Dehaene, S. (2004). Specialization within the ventral stream: The case for the visual word form area. NeuroImage, 22, 466476.

Cohen, L., Dehaene, S., Naccache, L., Lehericy, S., DehaeneLambertz, G., Henaff, M. A., \& Michel, F. (2000). The visual word form area: Spatial and temporal characterization of an initial stage of reading in normal subjects and posterior split-brain patients. Brain, 123, 291-307.

Dehaene, S., Jobert, A., Naccache, L., Ciuciu, P., Poline, J. B., Le BiHan, D., \& CoHEN, L. (2004). Letter binding and invariant recognition of masked words: Behavioral and neuroimaging evidence. Psychological Science, 15, 307-313.

Dehaene, S., Le Clec'H, G., Poline, J. B., Le Bihan, D., \& Cohen, L. (2002). The visual word form area: A prelexical representation of visual words in the fusiform gyrus. NeuroReport, 13, 321-325.

Dehaene, S., Naccache, L., Cohen, L., Le Bihan, D., Mangin, J. F., Poline, J. B., \& Riviere, D. (2001). Cerebral mechanisms of word masking and unconscious repetition priming. Nature Neuroscience, 4, 678-680.

Devlin, J. T., Moore, C. J., Mummery, C. J., Gorno-Tempini, M. L., Phillips, J. A., NopPeney, U., ET AL. (2002). Anatomic constraints on cognitive theories of category specificity. Neurolmage, 15, 675-685.

Downing, P. E., Jiang, Y., Shuman, M., \& Kanwisher, N. (2001). A cortical area selective for visual processing of the human body. Science, 293, 2470-2473. 
Epstein, R., \& KANwisher, N. (1998). A cortical representation of the local visual environment. Nature, 392, 598-601.

FARAH, M. J. (1990). Visual agnosia. Cambridge, MA: MIT Press.

Flowers, D. L., Jones, K., Noble, K., VanMeter, J., Zeffiro, T. A., Wood, F. B., \& EDEN, G. F. (2004). Attention to single letters activates left extrastriate cortex. NeuroImage, 21, 829-839.

Frackowiak, R., Friston, K., Frith, C., Dolan, R., \& Mazziotta, J. C. (1997). Human brain function. New York: Academic Press.

Gabrieli, J. D. E., Poldrack, R. A., \& Desmond, J. E. (1998). The role of left prefrontal cortex in language and memory. Proceedings of the National Academy of Sciences, 95, 906-913.

Garrett, A. S., Flowers, D. L., Absher, J. R., Fahey, F. H., Gage, H. D., KeYeS, J. W., ET AL. (2000). Cortical activity related to accuracy of letter recognition. NeuroImage, 11, 111-123.

GAUTHIER, I. (2000). What constrains the organization of the ventral temporal cortex? Trends in Cognitive Sciences, 4, 1-2.

Gauthier, I., \& Logothetis, N. (2000). Is face recognition not so unique after all? Cognitive Neuropsychology, 17, 125-142

Gauthier, I., Tarr, M. J., Anderson, A. W., Skudlarski, P., \& Gore, J. C. (1999). Activation of the middle fusiform "face area" increases with expertise in recognizing novel objects. Nature Neuroscience, $\mathbf{2}$, 568-573.

Gauthier, I., Tarr, M. J., Moylan, J., Skudlarski, P., Gore, J. C., \& ANDERSON, A. W. (2000). The fusiform "face area" is part of a network that processes faces at the individual level. Journal of Cognitive Neuroscience, 12, 495-504.

Gauthier, I., Wong, A. C.-N., Hayward, W. G., \& Cheung, S.-C. (2004). Font tuning associated with expertise in letter perception. Manuscript submitted for publication.

Genovese, C. R., Lazar, N. A., \& Nichols, T. (2002). Thresholding of statistical maps in functional neuroimaging using the false discovery rate. NeuroImage, $\mathbf{1 5}, 870-878$

Grill-Spector, K. (2003). The neural basis of object perception. Current Opinion in Neurobiology, 13, 159-166.

Grill-Spector, K., Kushnir, T., Edelman, S., Avidan, G., ItZChaK, Y, \& MALACH, R. (1999). Differential processing of objects under various viewing conditions in the human lateral occipital complex. Neuron, 24, 187-203

Hagoort, P., Indefrey, P., Brown, C., Herzog, H., Steinmetz, H., \& SeITz, R. J. (1999). The neural circuitry involved in the reading of German words and pseudowords: A PET study. Journal of Cognitive Neuroscience, 11, 383-398.

Hasson, U., Levy, I., Behrmann, M., Hendler, T., \& Malach, R. (2002). Eccentricity bias as an organizing principle for human highorder object areas. Neuron, 34, 479-490.

Ishai, A., Ungerleider, L. G., Martin, A., Schouten, J. L., \& HaXby, J. (1999). Distributed representation of objects in the human ventral visual pathway. Proceedings of the National Academy of Sciences, 96, 9379-9384

JAMES, K. H., \& GAUTHIER, I. (2005). Neural specialization for letters. Effects of writing, imagining, and perceiving letters. Manuscript in preparation

James, K. H., Martelli, M., James, T. W., Majaj, N. J., Pelli, D. G., \& GAUTHIER, I. (2004, April-May). fMRI reveals the role of the left fusiform gyrus in letter detection. Poster presented at the annual meeting of the Vision Sciences Society, Sarasota, FL.

Jobard, G., Crivello, F., \& Tzourio-Mazoyer, N. (2003). Evaluation of the dual route theory of reading: A metanalysis of 35 neuroimaging studies. NeuroImage, 20, 693-712.

Johnston, J. C., \& McClelland, J. L. (1974). Perception of letters in words: Seek not and ye shall find. Science, 184, 1192-1194.

Joseph, J. E., Gathers, A. D., \& PiPer, G. A. (2003). Shared and dissociated cortical regions for object and letter processing. Cognitive Brain Research, 17, 56-67.

Kanwisher, N. (2000). Domain specificity in face perception. Nature Neuroscience, 3, 759-763.

Kanwisher, N., Chun, M. M., \& McDermott, J. (1996). fMRI in individual subjects reveals loci in extrastriate cortex differentially sensitive to faces and objects. Investigative Ophthalmology \& Visual Science, 37, $\mathrm{S} 193$

Kanwisher, N., McDermott, J., \& Chun, M. M. (1997). The fusiform face area: A module in human extrastriate cortex specialized for face perception. Journal of Neuroscience, 17, 4302-4311.

Kircher, T. T., Brammer, M., Tous, A. N., Williams, S. C., \& McGuire, P. K. (2001). Engagement of right temporal cortex during processing of linguistic context. Neuropsychologia, 39, 798-809.

LaBerge, D., \& SAMUELS, S. J. (1974). Toward a theory of automatic information processing in reading. Cognitive Psychology, 6, 293-323.

Longcamp, M., Anton, J. L., Roth, M., \& Velay, J. L. (2003). Visual presentation of single letters activates a premotor area involved in writing. NeuroImage, 19, 1492-1500.

Malach, R., LeVy, I., \& Hasson, U. (2002). The topography of highorder human object areas. Trends in Cognitive Sciences, 6, 176-184

Malach, R., Reppas, J. B., Benson, R. R., Kwong, K. K., Jiang, H., KENNEDY, W. A., ET AL. (1995). Object-related activity revealed by functional magnetic resonance imaging in human occipital cortex. Proceedings of the National Academy of Sciences, 92, 8135-8139.

McCandliss, B. D., Cohen, L., \& Dehaene, S. (2003). The visual word form area: Expertise for reading in the fusiform gyrus. Trends in Cognitive Sciences, 7, 293-299.

MCClelland, J. L. (1977). Letter and configuration information in word identification. Journal of Verbal Learning \& Verbal Behavior, 16, 137-150.

MewhoRT, D. J. K. (1974). Accuracy and order of report in tachistoscopic identification. Canadian Journal of Psychology, 28, 383-398.

MonK, A. F., \& Hulme, C. (1983). Errors in proofreading: Evidence for the use of word shape in word recognition. Memory \& Cognition, 11, $16-23$

Moore, C. J., \& Price, C. J. (1999). Three distinct ventral occipitotemporal regions for reading and object naming. NeuroImage, 10, 181-192.

Murtha, S., Chertkow, H., Beauregard, M., \& Evans, A. (1999). The neural substrate of picture naming. Journal of Cognitive Neuroscience, 11, 399-423.

Ogawa, S., Menon, R. S., Tank, D. W., Kim, S. G., Merkle, H., EllerManN, J. M., \& UgurbiL, K. (1993). Functional brain mapping by blood oxygenation level-dependent contrast magnetic resonance imaging: A comparison of signal characteristics with a biophysical model. Biophysiology Journal, 64, 803-812.

Pelli, D. G., Farell, B., \& Moore, D. C. (2003). The remarkable inefficiency of word recognition. Nature, 423, 752-756.

Pillsbury, W. B. (1897). A study in apperception. American Journal of Psychology, 8, 315-393.

PolK, T. A., \& FARAH, M. J. (1995). Brain localization for arbitrary stimulus categories: A simple account based on Hebbian learning. Proceedings of the National Academy of Sciences, 92, 12370-12373.

PoLK, T. A., \& FARAH, M. J. (1998). The neural development and organization of letter recognition: Evidence from functional neuroimaging, computational modeling, and behavioral studies. Proceedings of the National Academy of Sciences, 95, 847-852.

PolK, T. A., \& FARAH, M. J. (2002). Functional MRI evidence for an abstract, not perceptual, word-form area. Journal of Experimental Psychology: General, 131, 65-72.

Polk, T. A., Stallcup, M., Aguirre, G. K., Alsop, D. C., D'Esposito, M., Detre, J. A., \& FARAH, M. J. (2002). Neural specialization for letter recognition. Journal of Cognitive Neuroscience, 14, 145159.

PrICE, C. J. (2000). The anatomy of language: Contributions from functional neuroimaging. Journal of Anatomy, 197, 335-359.

Price, C. J., \& Devlin, J. T. (2003). The myth of the visual word form area. Neurolmage, 19, 473-481.

Puce, A., Allison, T., Asgari, M., Gore, J. C., \& McCarthy, G. (1996). Differential sensitivity of human visual cortex to faces, letterstrings, and textures: A functional magnetic resonance imaging study. Journal of Neuroscience, 16, 5205-5215.

Pugh, K. R., Mencl, W. E., Jenner, A. R., Katz, L., Frost, S. J., Lee, J. R., ET AL. (2001). Neurobiological studies of reading and reading disability. Journal of Communication Disorders, 34, 479-492.

ReICHeR, G. M. (1969). Perceptual recognition as a function of meaningfulness of stimulus material. Journal of Experimental Psychology, 81, 274-280.

Rosch, E., Mervis, C. B., Gray, W. D., Johnson, D. M., \& Boyes- 
Braem, P. (1976). Basic objects in natural categories. Cognitive Psychology, 8, 382-439.

Rossion, B., Gauthier, I., Goffaux, V., Tarr, M. J., \& Crommelinck, M. (2002). Expertise training with novel objects leads to left lateralized face-like electrophysiological responses. Psychological Science, 13, 250-257.

Sanocki, T., Bowyer, K. W., Heath, M. D., \& Sarkar, S. (1998). Are edges sufficient for object recognition? Journal of Experimental Psychology: Human Perception \& Performance, 24, 340-349.

Tagamets, M. A., Novick, J. M., Chalmers, M. L., \& Friedman, R. B. (2000). A parametric approach to orthographic processing in the brain: An fMRI study. Journal of Cognitive Neuroscience, 12, 281-297.

Tarkiainen, A., Helenius, P., Hansen, P. C., Cornelissen, P. L., \& Salmelin, R. (1999). Dynamics of letter string perception in the human occipitotemporal cortex. Brain, 122, 2119-2131.

van Leeuwen, C., \& Lachmann, T. (2004). Negative and positive congruence effects in letters and shapes. Perception \& Psychophysics, 66, 908-925.
WheELER, D. D. (1970). Processes in word recognition. Cognitive Psychology, 1, 59-85.

Wong, A. C.-N., \& GAUTHIER, I. (2002). Visual expertise with different alphabet systems. Unpublished manuscript.

Wong, A. C.-N., Gauthier, I., Woroch, B., DeBuse, C., \& CurRAN, T. (2005). An early electrophysiological response associated with expertise in letter perception. Cognitive, Affective, \& Behavioral Neuroscience, 5, 306-318.

Wong, A. C.-N., Jobard, G., James, T. W., James, K. H., \& GaUTHIER, I. (2005). Expertise with characters in alphabetic and nonalphabetic writing systems engage the same occipitotemporal area. Manuscript submitted for publication.

(Manuscript received April 12, 2004; revision accepted for publication June 7, 2005.) 